\title{
Correlative X-ray and neutron tomography of root systems using cadmium fiducial markers
}

\author{
Thomas Clark ${ }^{1}$, Genoveva Burca ${ }^{2}$, Richard Boardman ${ }^{1}$, Thomas Blumensath ${ }^{1}$ \\ ${ }^{1}$ University of Southampton, Southampton, SO17 1BJ, UK \\ ${ }^{2}$ STFC, Rutherford Appleton Laboratory, ISIS Facility, Harwell, OX11 0QX, UK
}

Contact: tic3g13@soton.ac.uk, thomas.blumensath@soton.ac.uk

Keywords: X-ray, neutron, imaging, CT, computed tomography, registration, fiducial, crop science

\begin{abstract}
The interactions between plant roots and soil are an area of active research, particularly in terms of water and nutrient uptake. Since non-invasive, in vivo studies are required, tomographic imaging appears an obvious method to use, but no one imaging modality is well suited to capture the complete system. X-ray imaging gives clear insight to soil structure and composition, however water is comparatively transparent to X-rays and biological matter also displays poor contrast with respect to the pores between soil particles. Neutron imaging presents a complementary view where water and biological matter are better distinguished but the soil minerals are not imaged as clearly as they would be with $\mathrm{X}$-rays.
\end{abstract}

This work aims to develop robust methods for complementary X-ray/neutron tomographic imaging of plant root samples which should lead to new insight into water and nutrient transport in soil. The key challenges of this project are to develop experiments that will meet the requirements of both imaging modalities as well as the biological requirements of the plant samples and to develop ways to register a pair of reconstructed volume images of a sample that will typically have been produced with entirely separate facilities. The use of cadmium fiducial markers for registration has been investigated. Simulations were conducted to investigate the expected registration accuracy as the quantity and distribution of the markers varied. The findings of these simulations were then tested experimentally as plant samples were grown and imaged using neutrons with the IMAT instrument at ISIS Neutron and Muon Source at the STFC Rutherford Appleton Laboratory in Harwell, and with $\mathrm{X}$-rays at $\mu$-VIS X-ray Imaging Centre at the University of Southampton.

\section{Lay Description}

The interactions between plant roots and soil are an area of active research, particularly in terms of water and nutrient uptake. The samples used in this research are typically imaged so that they can be studied without digging up the roots and destroying the sample in the process. X-ray and neutron imaging techniques have both been used as each can show different materials within the sample. Since neither can show all the components of the system by itself, this work explores methods for combining scans of the same sample to give a more complete image of the system. In particular this work focusses on the use of fiducial markers as a strategy for preparing the samples in such a way that the resulting images can be aligned. The effectiveness of this method was tested in simulation and then in practice. The samples used within this work were imaged using neutrons on the IMAT 
instrument at ISIS Neutron and Muon Source at the STFC Rutherford Appleton Laboratory in Harwell, and with X-rays at $\mu$-VIS X-ray Imaging Centre at the University of Southampton.

\section{Introduction}

The human race is dependent upon plants to provide all food, either directly or indirectly. As the earth's population increases, a corresponding growth in crop yield is required. Recent estimates state that crop production will need to double by 2050 in order to keep pace with projected population growth [1], a target that is not predicted to be achieved by current growth projections [2]. Climate change will make this all the more difficult, in particular through reduced water availability and the drive to reduce fertiliser usage [3]. The green revolution is the name given to a period in the mid twentieth century when a number of scientific advancements, including the introduction of fertilisers and genetic modification, led to a tremendous gain in crop yields in a relatively short period of time [4]. The green revolution was primarily centred on the manipulation of the portion of the plant that is visible above the ground and roots were largely overlooked. The root system is central to plant functions such as water and nutrient uptake, anchorage and interaction with symbiotic organisms and so it has been recognised that root growth and development could be further exploited to maximise crop yield [5]. It has been suggested that the deployment of crops with more efficient water and nutrient uptake due to improved traits below the ground could lead to a second green revolution and help to address the world wide challenge of food security [6].

Non-invasive, in vivo studies of plant roots present a challenge that has traditionally been addressed through rhizotrons (containers that force $2 \mathrm{D}$ growth conformation with transparent windows for observation) or transparent, artificial growth media. Although widely used, these methods generally result in root systems that vary considerably from those grown in natural soil conditions [7]. X-ray computed tomography is the primary method for 3D imaging of root systems grown in soil but is not without its limitations [8]. While X-rays show the soil structure and composition well, they are not an ideal tool for imaging water distribution in particular since there is very little contrast between water, plant roots and any other biological soil constituents. None of these hydrogen-rich constituents are resolved clearly and as a result it is difficult to differentiate between them in the image data produced.

Neutron imaging offers a solution as many of the imaging techniques are similar but the mechanisms by which neutrons interact with matter are very different and hence different elements, in particular light elements such as hydrogen, provide strong contrast in a neutron image [9]. A number of experiments have shown neutron imaging to be well suited to showing water dynamics, where X-ray imaging would have struggled [10-15]. Neutron imaging has its own limitations however: it is slower, less readily available and produces images of lower resolution when compared to equivalent $X$-ray techniques. Just as X-ray imaging cannot provide a good representation of all the materials in a plant sample, neutron imaging struggles to show the soil minerals and structure clearly.

\section{Registration}

$\mathrm{Bi}$-modal datasets require registration to align the data from each modality. Although some cases have successfully used mutual information in the sample to achieve registration [16], in general it has been shown that, due to the complementary nature of the modalities, there is no guarantee that there will be similar local features in corresponding datasets (particularly with multi-phase images) and it is therefore difficult to find a good registration solution based on common features within a sample [9]. To overcome this difficulty, fiducial markers can be attached to a sample to aid in registration. A fiducial marker is an object placed within an image to be used as a point of reference. To register volumes using fiducial markers, at least three fiducial points must be selected in both the reference and target images so that the registration parameters can be found and a suitable transform applied. In the case of CT volume data, this is typically achieved through an affine transform. 
Inevitably, there will be error in the registration process. Maurer et al. defined three types of error that can occur when using fiducial points for registration [17]:

- Fiducial localisation error (FLE): the average error in locating the position of the fiducial points. (Figure 1 (A))

- Fiducial registration error (FRE): the root mean square (RMS) error between corresponding fiducial points after registration. (Figure 1 (B))

- Target registration error (TRE): the error between corresponding points other than the fiducial points after registration. (Figure 1 (C))

Fitzpatrick and West built on these definitions by providing expressions for the expected FRE and TRE errors in terms of the expected FLE and the set of fiducial points used (eq. 1) [18]. From this expression, Wang and Song define an equation (eq. 2) to relate TRE to a particular distribution of markers independently of FLE or FRE and propose a deterministic, optimisation method for determining the quantity and layout of markers to minimise TRE at a point of interest $r$ [19]. The distance from $r$ to each axis is donated as $d_{k}$, where $k=(1,2,3)$ and $f_{k}$ is the RMS distance of all fiducial points to the $k^{\text {th }}$ coordinate axis.

$$
\begin{gathered}
E\left\{T R E^{2}(r)\right\} \approx \frac{E\left\{F R E^{2}\right\}}{N-2}\left(1+\frac{1}{3} \sum_{k=1}^{3} \frac{d_{k}{ }^{2}}{f_{k}{ }^{2}}\right) \\
T R E_{M}(r)=\frac{1}{N-2}\left(1+\frac{1}{3} \sum_{k=1}^{3} \frac{d_{k}{ }^{2}}{f_{k}{ }^{2}}\right)
\end{gathered}
$$

\section{X-ray and neutron imaging equipment}

This project is a pathfinder application for the new IMAT instrument at ISIS Neutron and Muon Source. IMAT is a combined cold neutron imaging and diffraction instrument designed to take advantage of the second ISIS target station to provide neutron radiography, neutron tomography, energy-selective neutron imaging and spatially resolved diffraction scans [20]. The instrument construction was completed in 2016 and since then IMAT has been running its imaging configuration [21].

ISIS TS-2 is a short-pulse source which operates at $40 \mathrm{~kW}$ and delivers pulses at a rate of $10 \mathrm{~Hz}$. IMAT uses a cold $(20 \mathrm{~K})$, coupled liquid hydrogen moderator to slow the neutrons. A straight, $44 \mathrm{~m}$ supermirror neutron guide transports the neutrons from the target to the experimental area. Three choppers are placed within the guide to filter the beam. A TO chopper removes fast neutrons and gamma radiation, then a pair of double-disk choppers define the wavelength band to ensure there is no frame overlap between successive neutron pulses. At the end of the guide is a pinhole selector that allows the aperture diameter (D) to be varied between five values to define different $L / D$ ratios, where $L$ (the distance from the aperture to the sample) is $10 \mathrm{~m}$ [22]. This results in a total flight path of $56 \mathrm{~m}$ to the sample. Between the aperture and the sample, the beam travels through a $9 \mathrm{~m}$ evacuated flight tube and is shaped by five sets of jaws [23]. The sample is placed on a combined translation and rotation system that is rated for up to 1.5 tonnes.

IMAT has a variety of detectors but this work made use of IMAT's optical detector. This is based on a Zyla sCMOS 4.2 Plus camera in an optical camera box with a field of view varying between $50 \times 50$ $\mathrm{mm}^{2}$ and $200 \times 200 \mathrm{~mm}^{2}$ and acts as an integrating detector with a range of scintillators [24].

$\mu$-VIS' modified Nikon/X-Tek HMX (225kVp) was used to produce the X-ray data for this work. This is a customised, general purpose $\mathrm{X}$-ray $\mathrm{CT}$ and radiographic inspection system. It can take samples up to $300 \mathrm{~mm}$ in height although this is reduced to approximately $150 \mathrm{~mm}$ if the robotic sample exchanger is used. The $225 \mathrm{kVp} \mathrm{X}$-ray source can be configured for high resolution or high flux by 
using different anodes and uses a PerkinElmer PE1621 flat panel detector to capture the image. The detector is made up of a $2000 \times 2000$ matrix of amorphous silicon pixels with discrete gadolinium oxysulphide scintillators.

\section{Simulation}

In order to determine the best configuration of fiducial markers before imaging the samples, a simulation was conducted based on the method presented by Wang and Song [19]. The method was adapted to the case where the sample is cylindrical and the registration is to be optimised over the whole volume of the sample rather than at a single point of interest.

$\operatorname{TRE}_{M}(r)$ is a dimensionless quantity that relates the expectation value of TRE to that of FRE at a point $r$, for a given set of markers M. Potential marker locations were given by a grid of points on the surface of the cylinder. By evaluating $\operatorname{TRE}_{M}(r)$ for a set of points evenly distributed throughout the volume and taking the RMS value, an estimate of the TRE ${ }_{M}$ value is calculated for the whole volume. This means that TRE $\mathrm{M}_{M}$ gives a measure of how well two volumes can be registered with a set of markers $M$ independently of the FLE and FRE.

The simulation was run for $N=\{4, \ldots, 16\}$, where $N$ is the number of fiducial markers, to establish how many fiducial points should be used and how they should be distributed about the sample. Figure 2 shows how the value of TRE $\mathrm{M}_{M}$ falls as the number of fiducial markers is increased. The significance of each marker to the accuracy of the registration also falls quickly as $N$ is increased as shown in figure 3. This was calculated by taking the RMS average of the change in TRE $E_{M}$ when each marker is removed from $M$. This trend confirms that for a larger number of markers, each marker is less significant so less error is introduced if a marker cannot be accurately located or used for the registration.

Figure 4 shows the optimal distributions found by the simulation for $N=10,16$. A number of trends can be observed from the distributions of markers for different values of $N$ and applied to the general distribution of fiducial markers around a cylinder.

The first clear pattern is that the markers are distributed evenly between the very top and the very bottom of the sample - maximising the distance between the markers and the centre of the volume. This is a simple principle to apply when attaching markers to the sample and also has practical benefits for the imaging process since it means that the markers can be placed higher and lower than the soil in the sample tube. This means that areas of photon or neutron starvation, that could produce artefacts in the reconstructed data due to the markers, can be located away from the region of interest as the soil sample will not fill the very top and bottom of the sample tube.

Another clear pattern is that the markers are placed on a $180^{\circ}$ arc at the top and another corresponding arc at the bottom rather than, say, alternating between the top and bottom of the cylinder. The markers are also evenly distributed around the cylinder, ensuring that the centroid of the fiducial points is close to the centre of the sample.

Following these results from the optimisation algorithm a number of particular distributions were chosen to further evaluate these patterns. By evalutating distributions with an even distribution of markers between the top and bottom it was found that this generates very similar results. When compared to arcs at the top and bottom, with the same positions in $X$ and $Y$, the results were equal to 2 significant figures and the small variations at greater precision did not conclusively show either arrangement to be consistently better than the other for all values of $\mathrm{N}$. It seems reasonable that the simulation gives arcs because it begins the optimisation with a small number of markers and adds optimised markers iteratively up to the required total rather than started with $\mathrm{N}$ markers and attempting to redistribute them all.

The optimal arrangements found by the simulation were compared to random arrangements to see how significant the differences are. 1,500,000 unique, random arrangements were evaluated for 
each value of $\mathrm{N}$. Figure 5 shows the minimum, mean and maximum values from these tests as well as the optimal values (as seen in Figure 2). Figure 5a shows that a poor arrangement can increase the error by as much as an order of magnitude when very few markers are used. With higher numbers of markers however it becomes clear that the variation between good and bad arrangements becomes insignificant. Figure $5 \mathrm{~b}$ shows the same data in the range where $\mathrm{N}$ varies between 12 and 16 . It can be seen that the optimal solutions found by the simulation are better than any of the values found in the 1.5 million random arrnagements but not by a significant amount. On average, a random distribution of $\mathrm{N}+1$ markers will give better results than the optimal distribution of $\mathrm{N}$ markers.

Equation 1 states that the expectation of target registration error squared will be proportional to the FRE, the number of markers and the distribution of the markers. The simulation results show that with only four markers the TRE can be reduced to half of the FRE and that it can be reduced to less than a tenth of the FRE by using more than twelve points. Using a high number of markers makes a clear improvement to the result and also allows for the potential loss of a point without seeing a large drop in accuracy.

\section{Materials and Methods}

A set of samples were imaged using both X-ray and neutron tomography and then registered to test the proposed registration scheme and demonstrate the complementarity of the two modalities for further studies.

Cadmium was proposed as a suitable material for fiducial markers since it has a large attenuation coefficient for both neutrons and X-rays, allowing easy segmentation in either modality. An initial scan, to confirm the suitability of the sample tube and markers, showed that the cadmium produced significant artefacts, primarily due to beam hardening and scattering. It was decided that the artefacts could be reduced to a satisfactory level provided the marker size was minimised and there were sufficient variations in the height of the markers to avoid streaking between two markers in a slice. Smaller cadmium pieces were cut and a new sample tube was scanned to ensure these changes were sufficient.

Once the sample tube and marker configuration were shown to be suitable for imaging, a set of plants were grown and imaged using both IMAT and the HMX at to produce a set of complementary volumes that could be used to develop and test registration techniques. Twenty lupine seeds were placed in wet paper towels to germinate. After six days, eight seeds that had begun to sprout were selected and transferred to the sample tubes. New sample tubes were introduced for this experiment that were made from boron free quartz with an inner diameter of $14 \mathrm{~mm}$ and a wall thickness of $1.5 \mathrm{~mm}$. Each tube had a single fibreglass wick to draw water for the plant. Within the tube the wick was surrounded by $1 \mathrm{tsp}$ of sand with particle sizes between $1.18 \mathrm{~mm}$ and $0.6 \mathrm{~mm}$. Soil of the same particle size range was then used to fill the tube, covering the seed around $20 \mathrm{~mm}$ from the top. Each sample had a different quantity or arrangement of fiducial markers, as listed in Table 1. These were selected to allow the simulation results to be compared to measured data. Due to beamtime limitations, samples 2 and 6 were not scanned. The samples were left to grow for five days before the scans began. The neutron scans were conducted first over two days and the X-ray scans were collected over the following two days.

The neutron scans were conducted using the optical camera with a $135 \mathrm{~mm}$ lens, this gave a FOV of $60 \times 60 \mathrm{~mm}$. A $60 \mu \mathrm{m} \mathrm{ZnS/LiF} \mathrm{scintillator} \mathrm{with} \mathrm{a} \mathrm{surface} \mathrm{area} \mathrm{of} 90 \times 90 \mathrm{~mm}$ was used. The beam was shaped using the $40 \mathrm{~mm}$ pinhole and the jaws were set from $40 \mathrm{~mm}$ to $70 \mathrm{~mm}$ to match the beam profile to the FOV as closely as can be achieved without introducing artefacts. The samples were positioned $15 \mathrm{~mm}$ from the detector and 964 projections were taken with an exposure time of $30 \mathrm{~s}$ per projection. The projections were reconstructed using the filtered back projection algorithm in Octopus Reconstruction versions 8.9.3.4 and 8.9.4.2 [25]. The X-ray scans were conducted in the 
$\mathrm{HMX}$ at $80 \mathrm{keV}$ and $87 \mu \mathrm{A} .1571$ projections were taken with four frames and a 500 ms exposure time. The projections were reconstructed using Nikon CT Pro 3D version 2.2.5386.22184.

Once the scans were reconstructed, registration was attempted by segmenting the cadmium pieces and taking their centres as fiducial points, before finding and applying the affine transform to best match these points. The cadmium centres were located using a threshold segmentation in conjunction with FIJI's 3D Objects Counter [26]. An affine transform can be determined to match the two point sets. This is achieved by removing the translation and scaling differences by centring the two points sets and using orthogonal reduction before the rotation component is determined using Horn's algorithm [27]. Once the rotation has been found, the scaling and translation components can be determined easily [28]. After the transform was applied, the volumes were then cropped to matching dimensions. In order not to discard the higher resolution data in the X-ray scan, the neutron data was considered the target volume. As a result it was scaled and hence resampled at a higher resolution than IMAT could have achieved.

\section{Results}

Figures 6 and 7 show example slices and volumes from the reconstructed data collected using neutron and $\mathrm{X}$-ray imaging respectively.

Following the registration process, the fiducial markers were then resegmented in each modality and the new positions compared to give the FRE for each sample. This can be taken as a reasonable indicator of registration accuracy in the case of an affine transform. The FRE values are shown in Table 1. The mean FRE of sample 5 was so much higher than that of the other samples due to one marker which was misaligned by 18.5575 voxels. The mean FRE without taking that marker into account is only 4.5003, which is far closer to the typical FRE values produced by the other samples. This error was introduced as a result of a large FLE for this marker in the X-ray data due to an artefact. Figure 8 shows how the threshold segmentation detects an area far greater than that of the cadmium piece due to the artefacts surrounding it. This increase in the volume of the segmented marker pulls the measured centre away from the true value and perhaps more importantly away from the position found in the complementary modality to which it is to be matched.

Figure 9 shows a slice from sample 8 after registration. In addition to the combined data, each modality is shown separately. The side by side and overlaid comparison clearly shows the differences in contrast and signal to noise ratio between the two modalities but also the accuracy of the registration - in particular when observing the aluminium tape around the outside of the tube. This image also allows the complementarity to be seen clearly. Not only does the neutron data show the plant root with greater contrast than the $\mathrm{X}$-ray data but it shows some of the soil particles that appear in the X-ray data while omitting others. This means that the combined data can be used to infer information about the different materials making up soil particles which could not be distinguished using $\mathrm{X}$-rays alone.

\section{Discussion and Conclusions}

The data collected in this experiment demonstrates the suitability of X-ray and neutron tomography for multi-modal studies, particularly into plant soil systems. In addition we have shown that fiducial markers and the registration algorithms used allow the data from the two modalities to be registered accurately, overcoming a lack of clear mutual information in the sample, and it can be seen that more information can be taken by combining techniques than could be collected from either technique in isolation.

There is no correlation between the FRE of the samples and the number and distribution of fiducial markers used. For example, sample 1 has the second lowest FRE but had the worst set of fiducial markers since it had the fewest markers and their arrangement was close to co-linear. Sample 5 had the greatest number of markers and therefore had the best set of fiducial points, but it showed the worst FRE of all the samples. The product of the FRE and TRE ${ }_{M}$ values (Table 1) gives an estimate for 
the TRE for each sample. The variation FRE is far more significant in this calculation than that of TRE which shows that being able to accurately image and locate the markers is more important to the overall registration accuracy than having the markers ideally distributed.

The higher FRE of sample 5 indicates that artefacts in the scans which affect the segmentation of the fiducial markers are the greatest source of error in registration (fig 8). Cadmium's high attenuation introduces artefacts and these have been shown to have a negative impact on the registration accuracy such that the effect of marker arrangement could not be tested in detail. This suggests that while it can be used as a fiducial marker material, it is not ideal. A material that would attenuate both X-rays and neutrons less would result in reduced artefacts, leading to not only better scan data but more accurate registration since large FLE values as a result of image artefacts have been shown to be the primary contributor to FRE in the registered data.

Further studies are being planned that will test the suitability of other materials, such as borosilicate as fiducial markers.

Movement and other changes in the samples can be observed in the registered results, as a result of the time and travel between the two scans of each sample. In particular, the seedlings begin to droop and the top layers of soil can move considerably between the two scans. This will be addressed in future studies by booking time to conduct both scans at the same site with minimal delays between them. In addition, these studies will integrate synchrotron X-ray imaging which will improve the resolution of the data and allow more complex imaging techniques to be introduced.

\section{References}

1. Tilman, D., et al., Global food demand and the sustainable intensification of agriculture. Proceedings of the National Academy of Sciences, 2011. 108(50): p. 20260-20264.

2. Ray, D.K., et al., Yield Trends Are Insufficient to Double Global Crop Production by 2050. PLOS ONE, 2013. 8(6): p. e66428.

3. Atkinson, J.A., et al., Uncovering the hidden half of plants using new advances in root phenotyping. Current Opinion in Biotechnology, 2019. 55: p. 1-8.

4. Bishopp, A. and J.P. Lynch, The hidden half of crop yields. Nature Plants, 2015. 1: p. 15117.

5. Herder, G.D., et al., The roots of a new green revolution. Trends in Plant Science, 2010. 15(11): p. 600-607.

6. Lynch, J.P., Roots of the Second Green Revolution. Australian Journal of Botany, 2007. 55(5): p. 493-512.

7. Kuijken, R.C.P., et al., Root phenotyping: from component trait in the lab to breeding. Journal of Experimental Botany, 2015. 66(18): p. 5389-5401.

8. Mairhofer, S., et al., RooTrak: Automated Recovery of Three-Dimensional Plant Root Architecture in Soil from X-Ray Microcomputed Tomography Images Using Visual Tracking. Plant Physiology, 2012. 158(2): p. 561-569.

9. Kaestner, A.P., et al., Bimodal Imaging at ICON Using Neutrons and X-rays. Physics Procedia, 2017. 88: p. 314-321.

10. Menon, M., et al., Visualization of root growth in heterogeneously contaminated soil using neutron radiography. European Journal of Soil Science, 2007. 58(3): p. 802-810.

11. Matsushima, U., et al., Estimation of water flow velocity in small plants using cold neutron imaging with D2O tracer. Nuclear Instruments and Methods in Physics Research Section A: Accelerators, Spectrometers, Detectors and Associated Equipment, 2009. 605(1-2): p. 146149.

12. Moradi, A.B., et al., Three-dimensional visualization and quantification of water content in the rhizosphere. New Phytologist, 2011. 192(3): p. 653-663.

13. Warren, J.M., et al., Neutron imaging reveals internal plant water dynamics. Plant and Soil, 2013. 366(1-2): p. 683-693. 
14. Zarebanadkouki, M., Y.X. Kim, and A. Carminati, Where do roots take up water? Neutron radiography of water flow into the roots of transpiring plants growing in soil. New Phytol, 2013. 199(4): p. 1034-44.

15. Totzke, C., et al., Capturing 3D Water Flow in Rooted Soil by Ultra-fast Neutron Tomography. Sci Rep, 2017. 7(1): p. 6192.

16. Kim, F., et al., Characterizing partially saturated compacted-sand specimen using 3D Image registration of high-resolution neutron and $X$-ray tomography. Journal of Computing in Civil Engineering, 2014. 29(6): p. 04014096.

17. Maurer, C.R., et al., Registration of head volume images using implantable fiducial markers. IEEE Transactions on Medical Imaging, 1997. 16(4): p. 447-462.

18. Fitzpatrick, J.M., J.B. West, and C.R. Maurer, Predicting error in rigid-body point-based registration. IEEE Transactions on Medical Imaging, 1998. 17(5): p. 694-702.

19. Wang, M. and Z. Song, Improving target registration accuracy in image-guided neurosurgery by optimizing the distribution of fiducial points. The International Journal of Medical Robotics and Computer Assisted Surgery, 2009. 5(1): p. 26-31.

20. Kockelmann, W., et al., IMAT - A New Imaging and Diffraction Instrument at ISIS. Physics Procedia, 2013. 43(Supplement C): p. 100-110.

21. BURCA, G., et al., Exploring the potential of neutron imaging for life sciences on IMAT. Journal of Microscopy. $\mathbf{0}(0)$.

22. Burca, G., et al., Modelling of an imaging beamline at the ISIS pulsed neutron source. Journal of Instrumentation, 2013. 8(10): p. P10001-P10001.

23. Kockelmann, W., et al., Status of the Neutron Imaging and Diffraction Instrument IMAT. Physics Procedia, 2015. 69: p. 71-78.

24. Finocchiaro, V., et al., The autofocusing system of the IMAT neutron camera. Review of Scientific Instruments, 2013. 84(9): p. 093701.

25. Dierick, M., B. Masschaele, and L. Van Hoorebeke, Octopus, a fast and user-friendly tomographic reconstruction package developed in LabView ${ }^{\circledast}$. Measurement Science and Technology, 2004. 15(7): p. 1366.

26. Schindelin, J., et al., Fiji: an open-source platform for biological-image analysis. Nat Meth, 2012. 9(7): p. 676-682.

27. Horn, B.K., Closed-form solution of absolute orientation using unit quaternions. JOSA A, 1987. 4(4): p. 629-642.

28. Qu, J., L. Gong, and L. Yang, A 3D point matching algorithm for affine registration. International Journal of Computer Assisted Radiology and Surgery, 2011. 6(2): p. 229-236.

29. West, J.B., et al., Fiducial Point Placement and the Accuracy of Point-based, Rigid Body Registration. Neurosurgery, 2001. 48(4): p. 810-817. 
Figure 1. The three types of registration errors: FLE, FRE and TRE. Based on Figure 1 of [29].

Figure 2. The change in target registration error as the number of fiducial points is increased.

Figure 3. The average contribution of a single marker to the target registration accuracy as the number of markers increases.

Figure 4. The optimal fiducial marker distributions suggested by the simulation for $N=10$ (top) and $N$ $=16$ (bottom).

Figure 5. A comparison of the optimal marker distributions to the mean, maximum and minimum $T R E_{M}$ values found in 1,500,000 random trials. 5 a (top) shows the full range of $N$ values. $5 b$ (bottom) shows $N=12-16$.

Figure 6. A slice and partial volume render from the neutron scan of sample 7.

Figure 7. A slice and partial volume render from the X-ray scan of sample 8.

Figure 8. Sample 5 had the worst registration accuracy despite having the most fiducial markers. This figure shows how an artefact prevents accurate segmentation (segmentation shown in red) of a cadmium piece, creating a large FLE for the corresponding fiducial marker.

Figure 9. A slice from sample 8 with the X-ray data on the left and in red and the neutron data on the right and in green. This slice shows the match of a fiducial marker, the accuracy of the registration and the complementarity of the modalities.

Table 1. The fiducial marker arrangements, FRE measurements and TRE $E_{M}$ values for each of the sample tubes that was scanned.

Figure 1

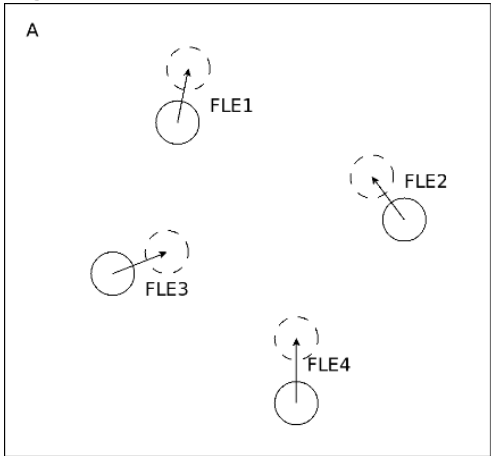

Figure2
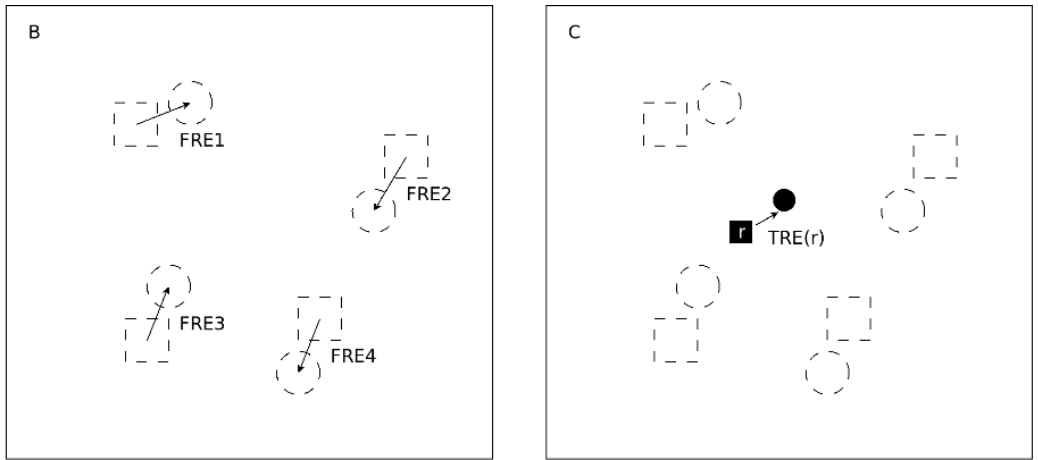
Figure 2

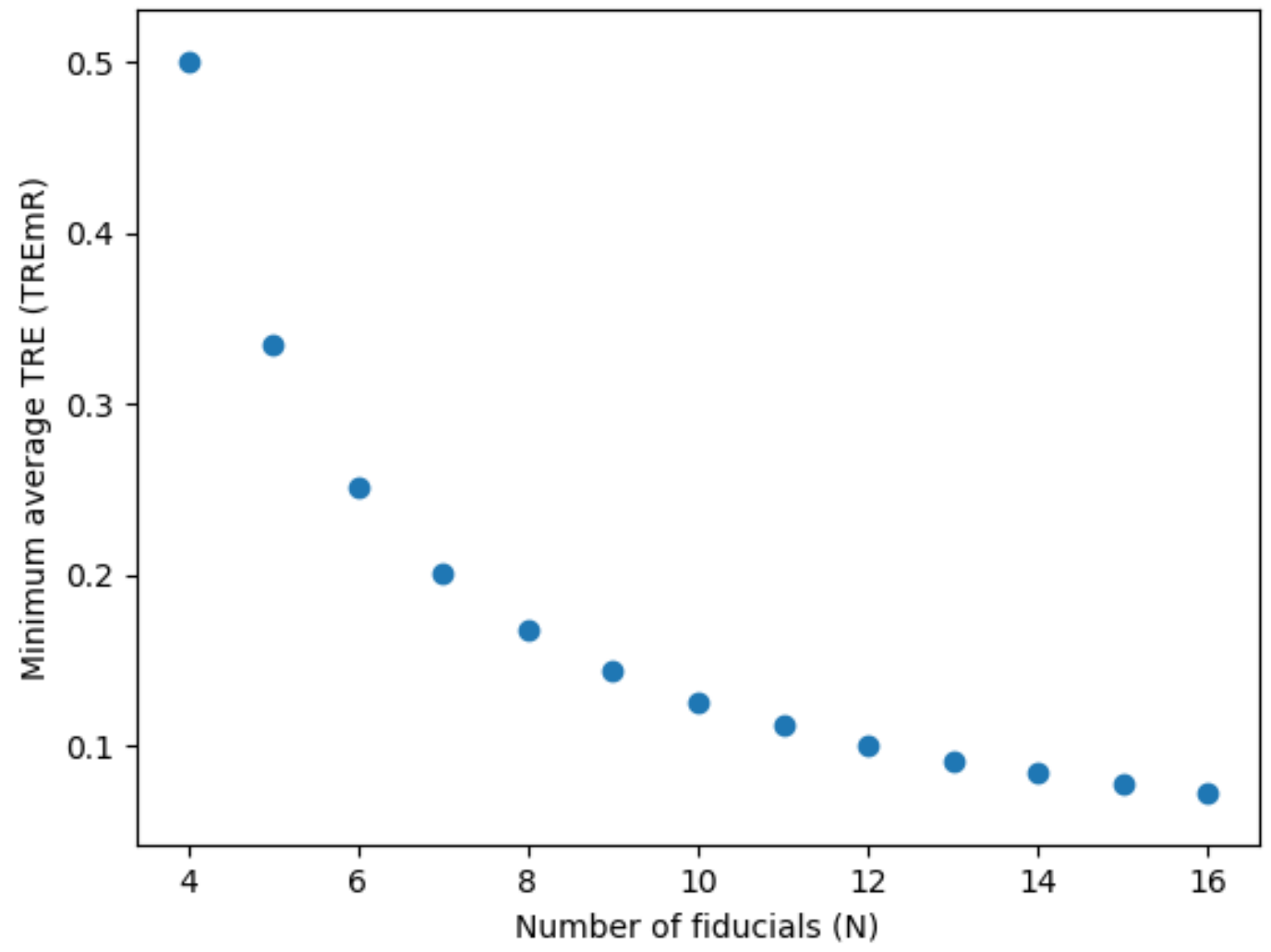


Figure 3

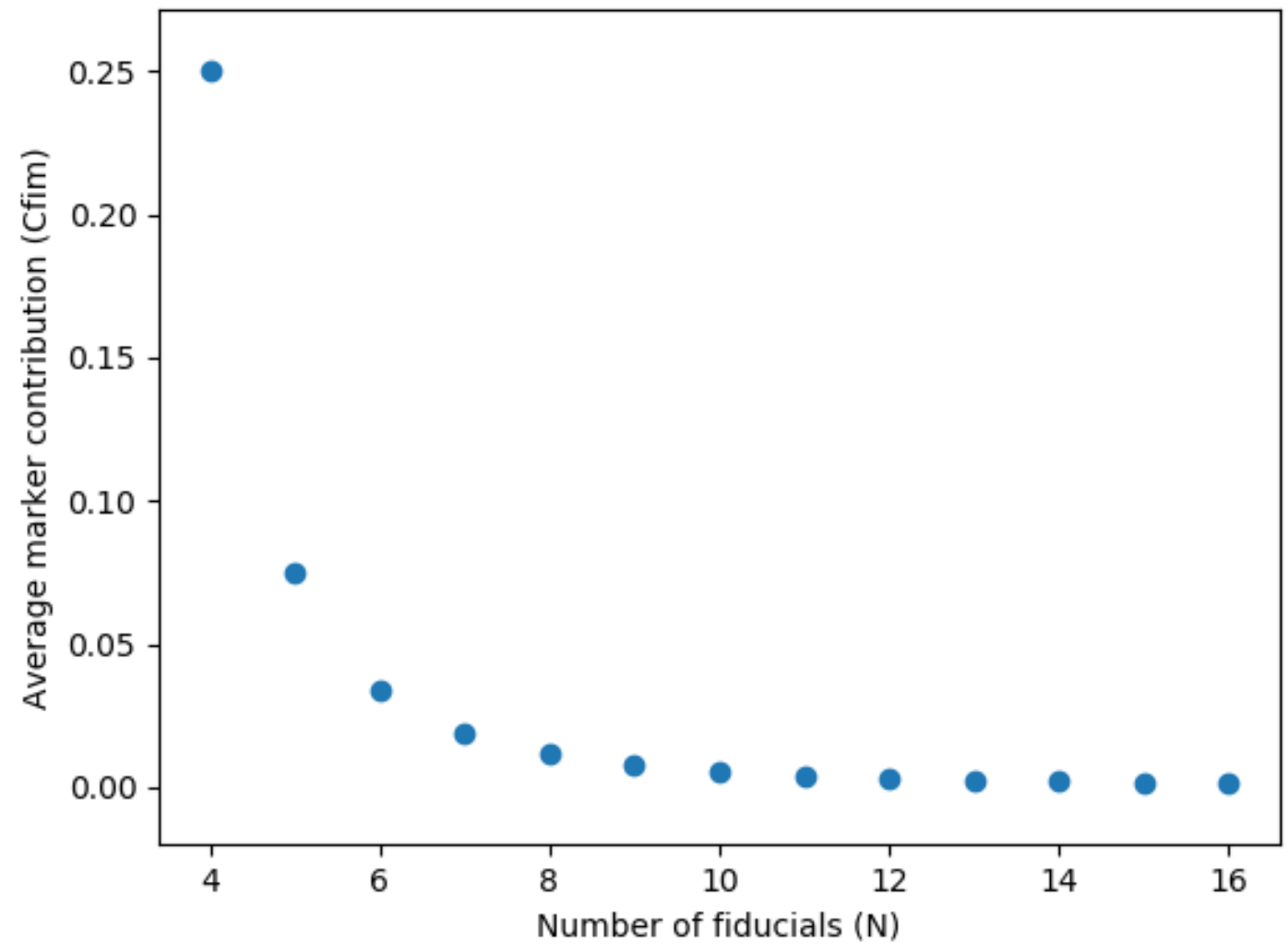


Figure 4
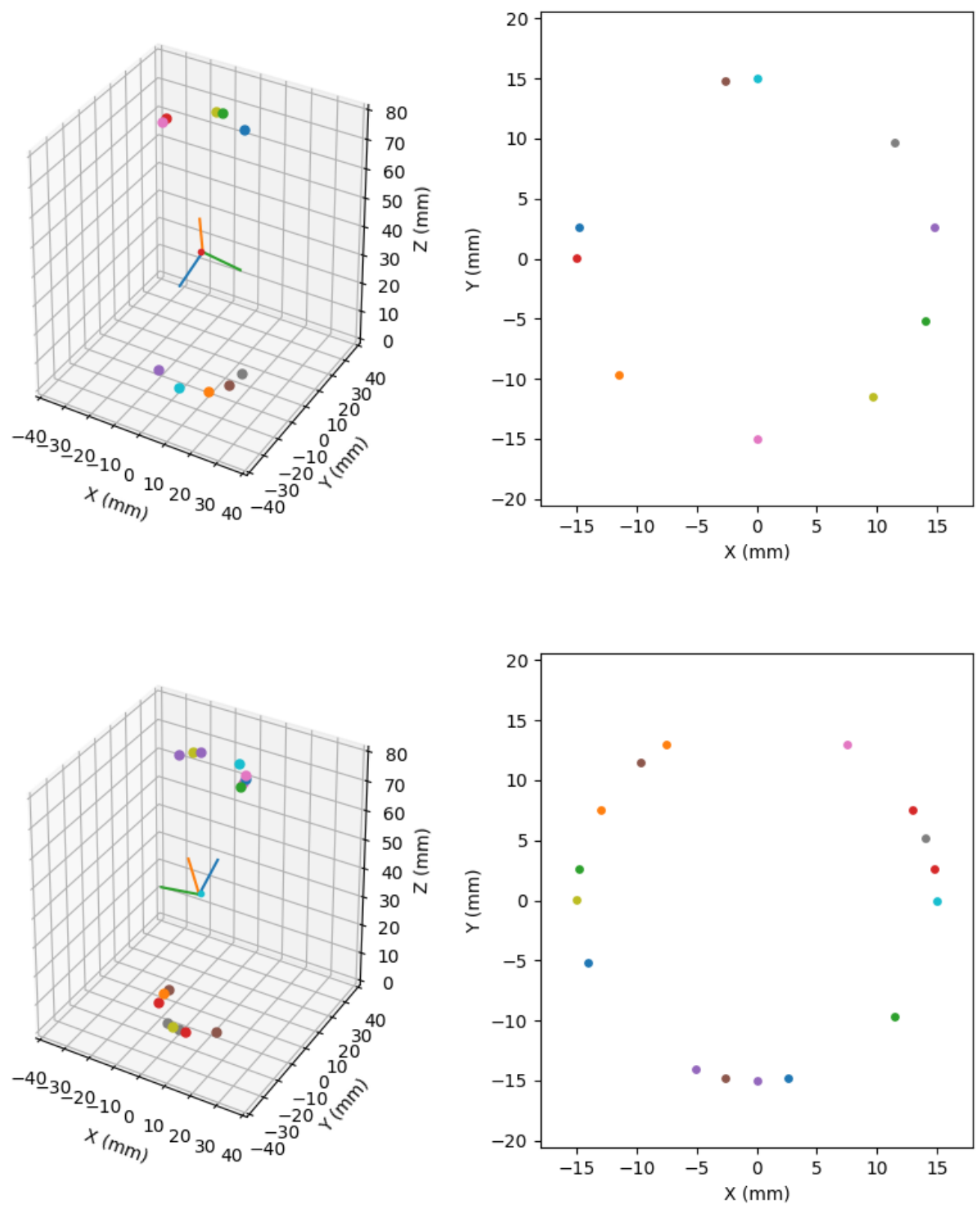
Figure 5
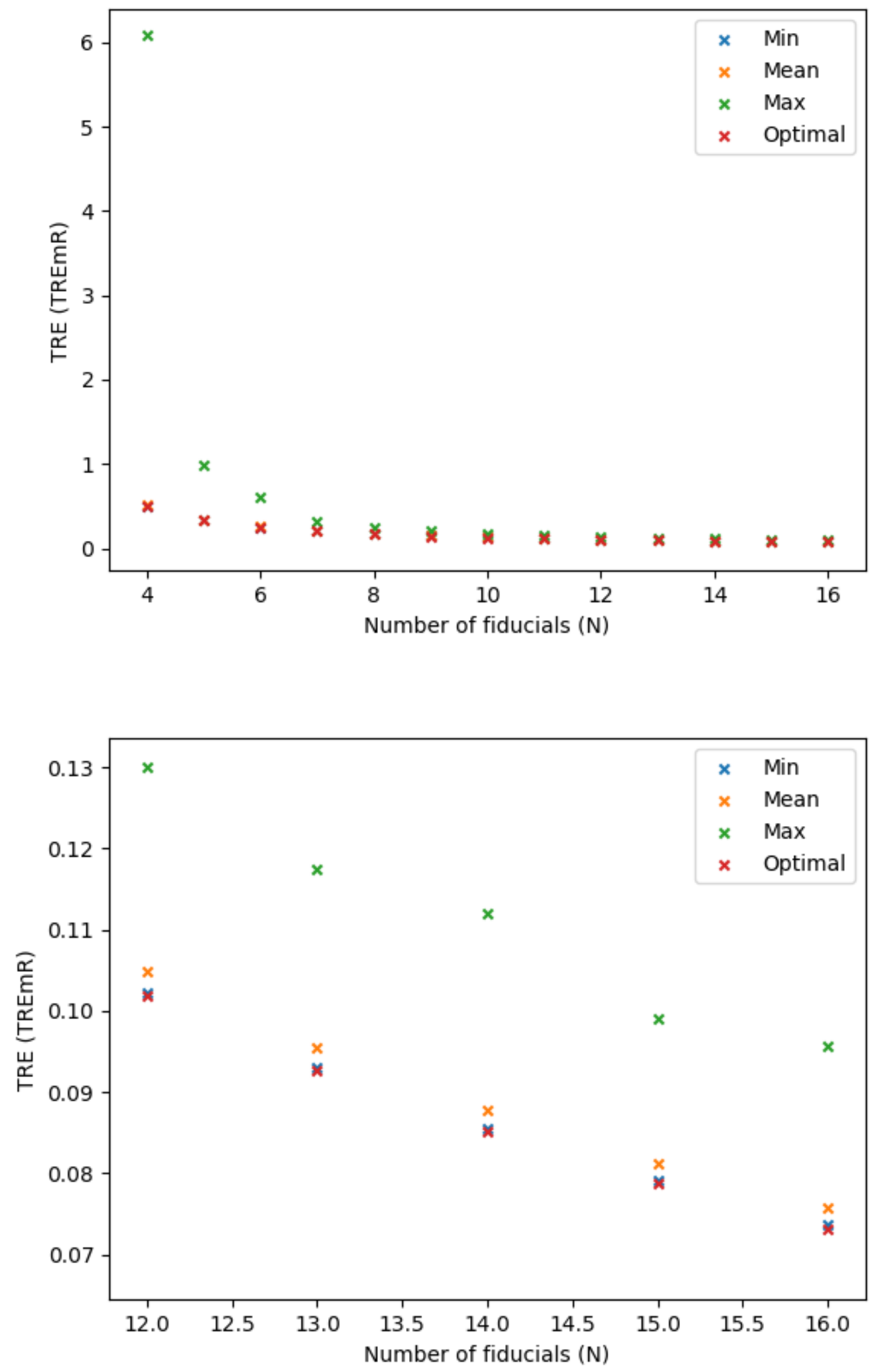
Figure 6

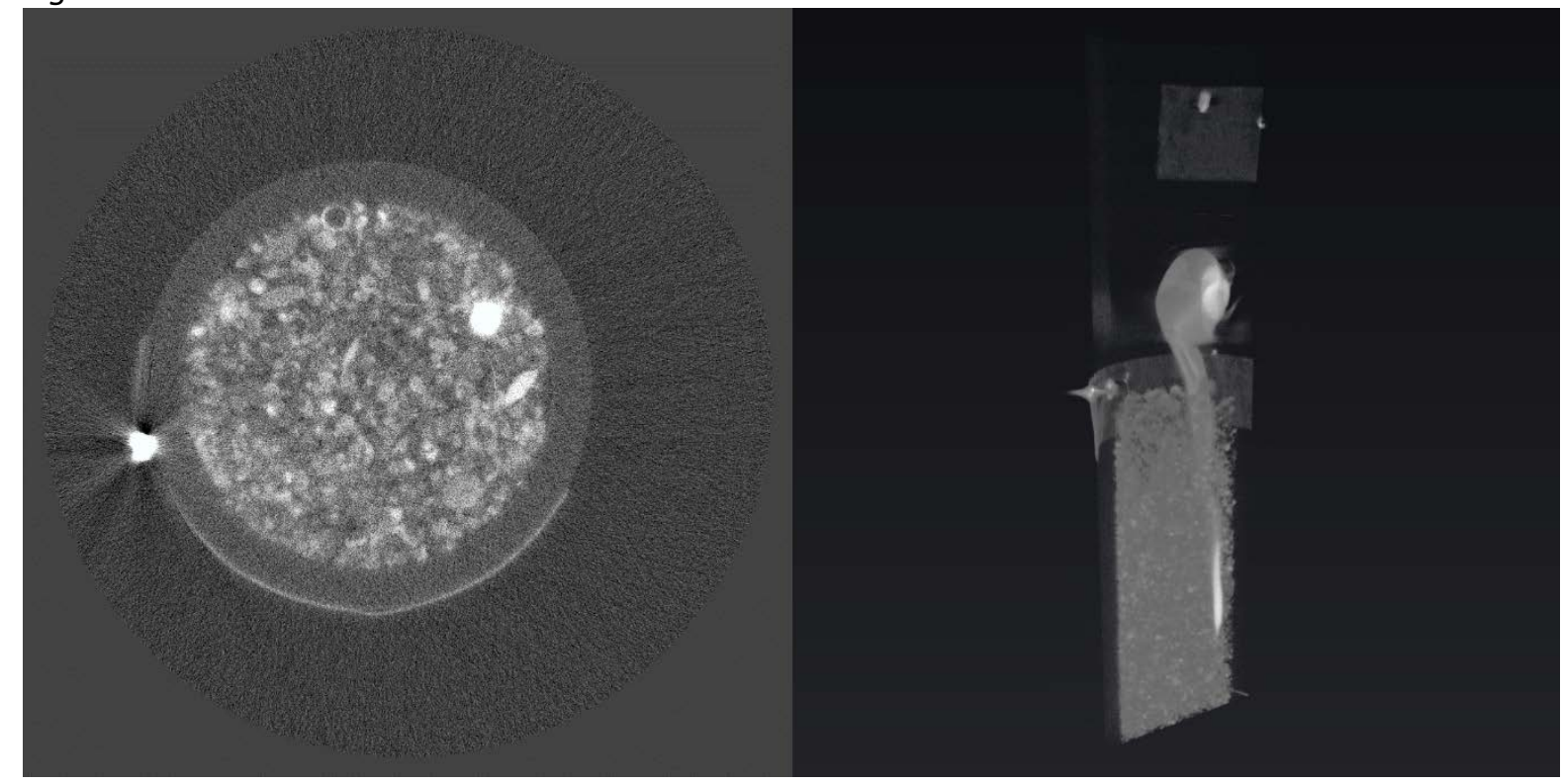

Figure 7

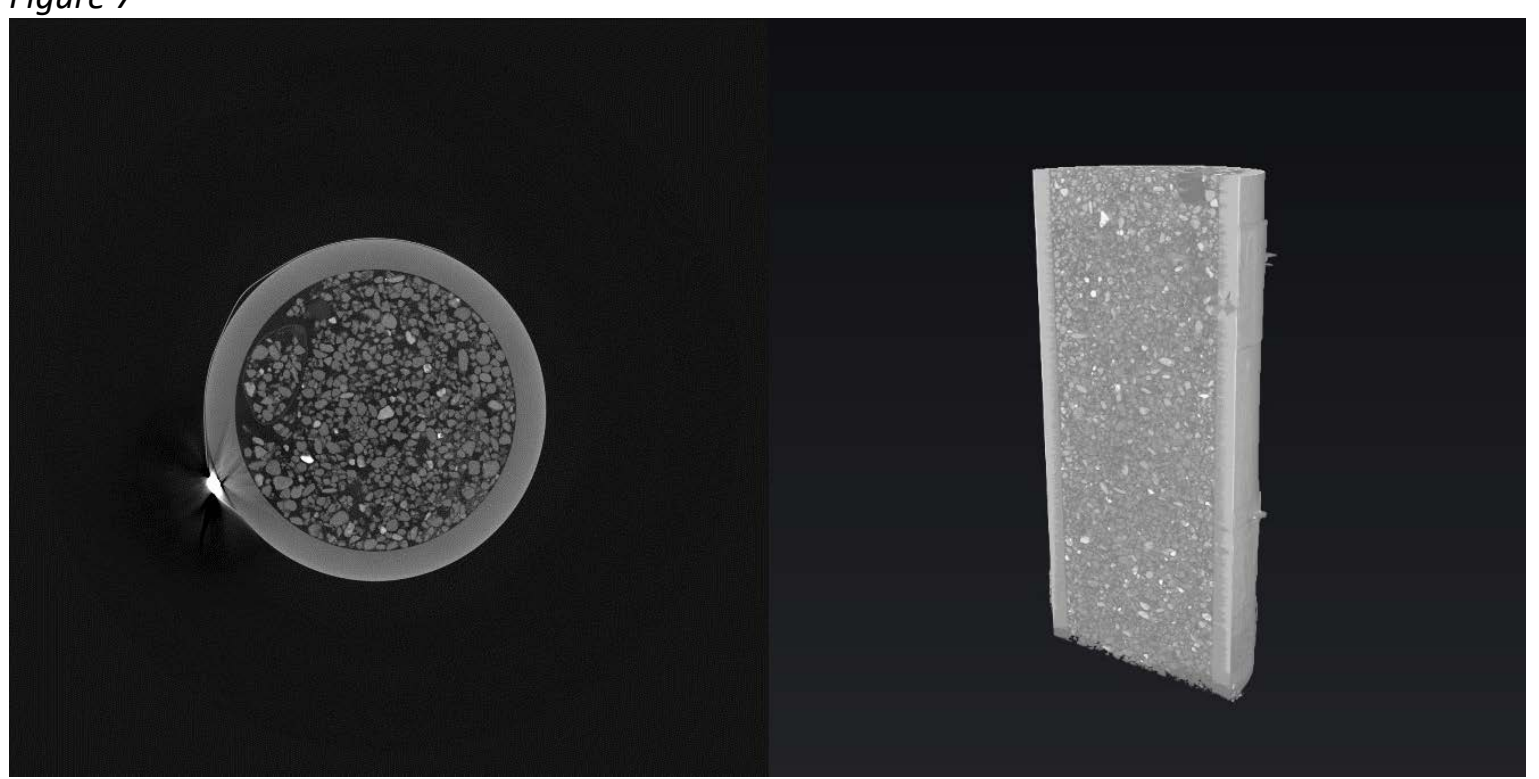




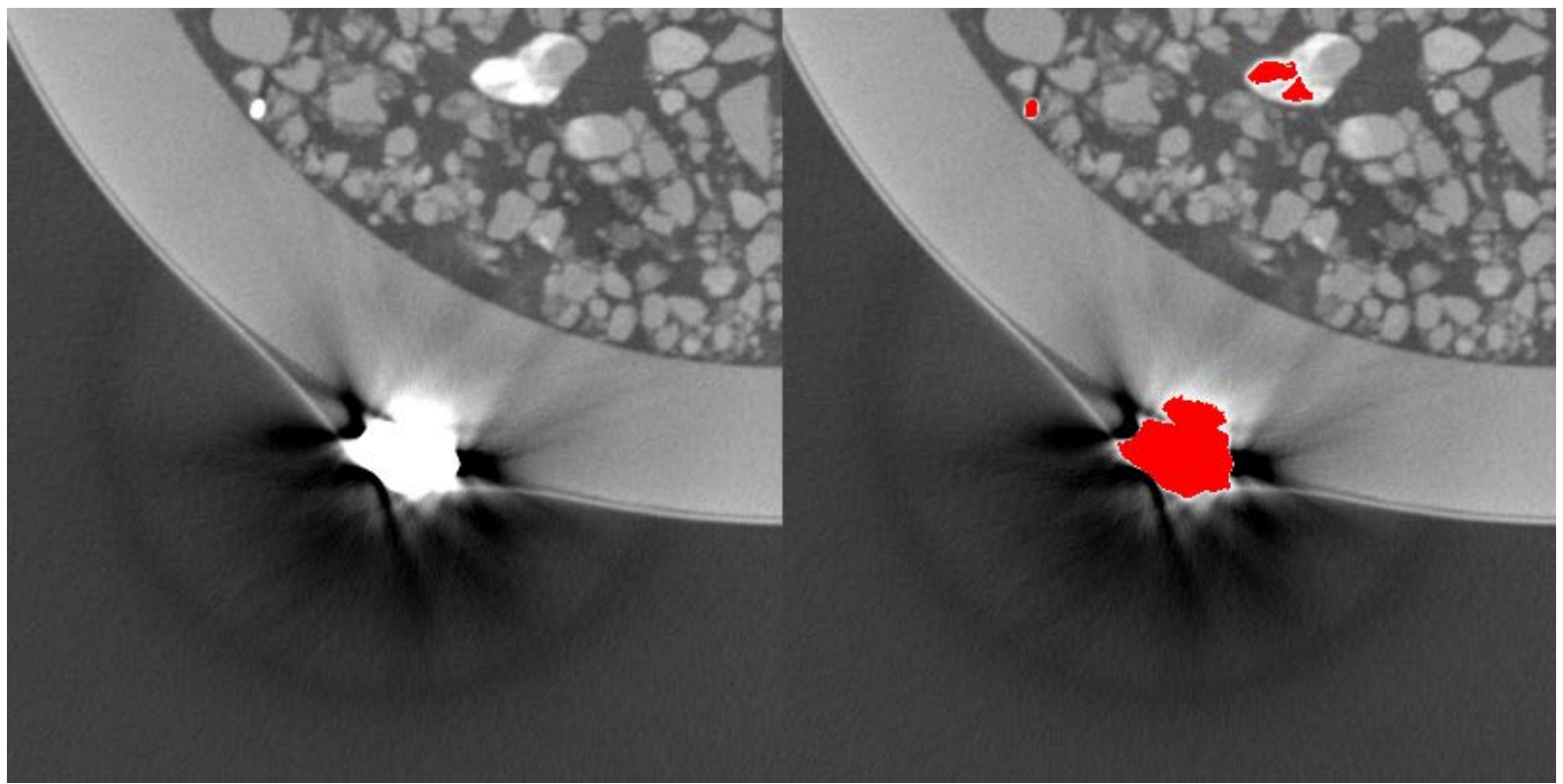

Figure 9

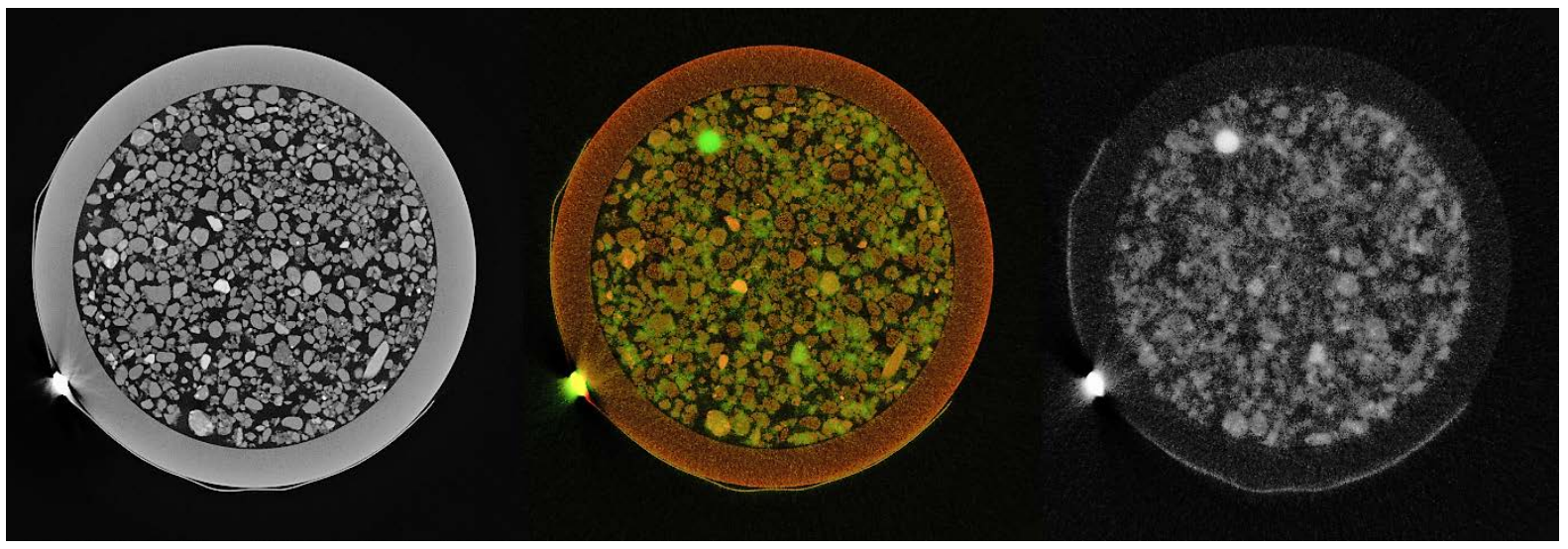

Table 1

\begin{tabular}{|c|c|c|c|c|}
\hline SAMPLE & FIDUCIAL ARRANGEMENT & FRE & $\mathrm{TRE}_{\mathrm{M}}$ & $\operatorname{TRE}\left(F R E \times T^{T R E} E_{M}\right)$ \\
\hline 1 & 5 markers in a vertical line & 2.6618 & 0.3334 & 0.8874 \\
\hline 3 & $\begin{array}{c}6 \text { markers in arcs at the top and bottom of } \\
\text { the FOV }\end{array}$ & 6.4916 & 0.2500 & 1.6229 \\
\hline 4 & $\begin{array}{c}8 \text { markers in arcs at the top and bottom of } \\
\text { the FOV }\end{array}$ & 2.0335 & 0.1667 & 0.3390 \\
\hline 5 & $\begin{array}{c}10 \text { markers in arcs at the top and bottom of } \\
\text { the FOV }\end{array}$ & 11.0246 & 0.1250 & 1.3781 \\
\hline 7 & $\begin{array}{c}9 \text { markers in arcs at the top, middle and } \\
\text { bottom of the FOV }\end{array}$ & 4.3284 & 0.1429 & 0.6185 \\
\hline 8 & $\begin{array}{c}9 \text { markers in arcs at the top, middle and } \\
\text { bottom of the FOV }\end{array}$ & 2.8543 & 0.1429 & 0.4079 \\
\hline
\end{tabular}

Table 1. The fiducial marker arrangements, FRE measurements and TRE $E_{M}$ values for each of the 